\title{
EDITORIAL
}

\section{Cell Replacement Therapy: Helping the Brain to Repair Itself}

\section{INTRODUCTION}

The interest in cell replacement therapies for human CNS disorders dates back to the late 1970s, when it was demonstrated that intrastriatal grafts of fetal mesencephalic tissue, rich in dopamine neurons, induced functional recovery in rats with neurotoxin-induced lesions of the nigrostriatal dopaminergic system. These observations provided the first evidence that neurons implanted into the adult brain could reverse behavioral deficits in an animal model of a human neurological disorder, Parkinson's disease. Based on a bulk of experimental data in rodents and nonhuman primates, the first clinical trials with neural transplantation in Parkinson's disease were initiated in 1987. Successful studies in animal models of Huntington's disease led to the first attempts with fetal striatal grafts in Huntington's disease patients in the late 1990s. In addition, neural transplantation has also been applied clinically, with varying degree of scientific foundation, in other disorders such as stroke and epilepsy, and in patients with spinal cord injury.

The basic principle of cell therapy is quite simple: restoration of function lost as a result of damage or disease in the CNS by the replacement of dead cells with new healthy ones. Given the complexity of both the structure and function of human brain and spinal cord, this prospect may seem remote. In fact, until 25 years ago, the possibility of using cell replacement for CNS repair was regarded as science fiction. Today, cell therapy is a realistic therapeutic possibility. Extensive animal experimental studies performed over the last two decades have shown that neuronal replacement and partial reconstruction of neuronal circuitry is indeed feasible. If this approach can be made to work in the human nervous system, cell therapy could provide radical new treatments for severe neurological disorders.

These emerging prospects raise a number of scientific and ethical concerns. First, the rationale for the application of cell therapy may not be equally good for all types of brain damage, and for some more complex CNS disorders the usefulness of the cell repair strategy remains highly speculative. Secondly, the rush to apply cell therapies in patients may lead to scientifically ill-founded clinical trials without sufficient support of rigorous preclinical research.

\section{PIONEERING STUDIES: PARKINSON'S AND HUNTINGTON'S DISEASE}

Neural transplantation is most likely to work for those types of neurons that have relatively nonspecific modulatory actions and whose function does not critically depend on complex and precise, patterned connectivity. The nigrostriatal dopamine neurons are good examples of this type. These neurons, which are located in the midbrain substantia nigra, provide a dense, diffuse innervation of one of the principal motor control centers of the forebrain, the striatum. Dopamine released from the nigrostriatal terminals acts in a tonic, level-setting manner to regulate motor behavior. Experimental studies have shown that lesions of the nigrostriatal dopamine neurons induce a profound akinetic state that, at least in part, is due to an increased threshold for activation of the striatal output system and initiation of movement. Neural transplantation in Parkinson's disease is based on the idea that dopamine-producing cells implanted into the denervated striatum might be able to substitute for those mesencephalic dopamine neurons that have been lost as a consequence of the disease process.

In rodent and primate models of Parkinson's disease, intrastriatal transplants of fetal nigral neurons have been shown to reverse or ameliorate impairments in both drug-induced and spontaneous motor behaviors induced by damage to the nigrostriatal system. The grafted nigral neurons re-establish a functional dopamine innervation of the previously denervated striatal target. They are spontaneously active and release dopamine in an impulse-dependent manner at both synaptic and nonsynaptic sites, although they are largely independent of any regulatory afferent inputs. The grafted dopamine neurons may function either by a "pharmacological" type of action, whereby the released dopamine is able to diffuse over sufficient distances to activate the denervated striatal receptors, and/or via functional reinnervation of the denervated target neurons by the outgrowing axons of the implanted neuroblasts, which allows released dopamine to exert its action at defined synaptic sites.

In patients suffering from Parkinson's disease, results from open-label clinical studies performed over the last 15 years convincingly show that transplanted fetal human midbrain dopamine neurons, taken at a stage of development when they have started to express their dopaminergic phenotype, can survive and function over a 
long time in the striatum, i.e., in brains affected by an ongoing disease process. Increased ${ }^{18} \mathrm{~F}$-fluorodopa uptake (i.e., dopamine synthesis and retention) in the areas reinnervated by the grafted cells has been maintained for at least 6-10 years in several of the patients studied the longest, and consistent with the imaging data, good survival of grafted dopamine neurons and extensive reinnervation of the surrounding host striatum have been demonstrated in several autopsy cases. Although significant long-term clinical benefit has been obtained in some of the transplanted patients, it remains unclear whether transplantation of fetal dopamine neurons can be developed into an effective and clinically competitive therapy.

In Huntington's disease, several open-label clinical trials have been performed using transplants of striatal primordia, obtained from the ganglionic eminences of the developing forebrain from either human or porcine fetal donors. Although initial reports described signs of improvements in parameters of motor function and cognition, it is still too early to determine whether any of these changes are attributable to the function of the transplanted cells. The best indications that fetal striatal transplants indeed may work in the larger and more complex primate brain come from studies in monkeys, which show that striatal transplants can induce significant recovery in both motor and cognitive functions in animals with toxin-induced destruction of the striatum, i.e., in the best primate models of Huntington's disease available today.

Experiments in rodents have shown that transplants of fetal striatal neurons can restore GABA synthesis and release within both the lesioned striatum and the adjacent, denervated globus pallidus. More importantly, however, the implanted striatal primordia develop striatum-like structures at the site of implantation, and part of the cells develop into fully differentiated striatal projection neurons. These cells, which are of the medium-size densely spiny type, grow to establish normal synaptic contracts with the output neurons in the globus pallidus, and they receive appropriate afferent synaptic inputs from thalamus, cortex, and substantia nigra. Thus, there is good evidence that transplanted fetal striatal neurons can reconstruct at least some critical elements of the damaged striatal circuitry in animal models of Huntington's disease. The results obtained in animal experimental studies are thus quite promising. Additional long-term clinical trials are needed to clarify whether these preclinical animal data can be translated into an effective therapy for Huntington's disease. In this context, it should be emphasized that it is a serious dilemma that the animal models of neurodegenerative diseases we have available today do not accurately reproduce the pathophysiology of the human conditions. For this reason, it is difficult to know whether results obtained in animal experimental studies will hold up when the same procedures are tested clinically.

\section{STEM CELLS}

Although the clinical studies with fetal tissue seem to provide proof-of-principle for the cell-replacement strategy, at least in Parkinson's disease and possibly also in Huntington's disease, it is obvious that other sources of cells are needed if cell-based approaches should ever become clinically competitive. Currently, much interest is focused on the possibility that stem cells or their derivatives could solve the problems associated with the use of fetal tissue grafts, e.g., poor availability and standardization of the cell material. Stem cells are immature cells with prolonged self-renewal capacity and, depending on their origin, an ability to mature into multiple cell types. Recent progress shows that neurons suitable for transplantation can be generated from stem cells in culture, and that the adult brain produces new neurons from its own stem cells in response to injury.

From a clinical perspective, the development of stem cell-based therapies for brain diseases is still at an early stage. Many basic issues remain to be solved. We do not know what is the best stem cell source, and research on embryonic stem cells and neural stem cells from fetal or adult brain, or cells derived from other tissues, should therefore be performed in parallel. We need to understand how to control stem cell proliferation and differentiation into specific cell types, induce their integration into neural networks, and optimize the functional recovery in animal models closely resembling the human disease. The regenerative potential of the self-repair mechanisms of the brain is virtually unexplored. The functional properties of the new neurons produced from endogenous stem cells and their ability to form connections should be determined. We should design strategies to deliver molecules that improve the yield of new functional neurons and other cells in the damaged area.

\section{CURRENT STATUS AND FUTURE PERSPECTIVES}

In this issue of NeuroRx ${ }^{\circledR}$, the current status of research in cell therapy is described for five different diseases for which this approach has already been tested clinically: Parkinson's disease, Huntington's disease, stroke, demyelinating disorders, and spinal cord injury. The contributions of Lindvall and Björklund, Dunnett and Rosser, Savitz and collaborators, Rice and collaborators, and Reier give an overview of the results from the clinical trials performed so far and their animal experimental basis as well as an overview of ongoing basic and clinical research. The authors also discuss the obstacles and problems that need to be overcome for the additional 
development of clinical cell therapy in the respective disease, i.e., they outline road maps for translational research that may lead to successful application of cell therapy in patients.

The second part of this issue deals with three topics of general importance for cell therapy and brain repair, i.e., the intrinsic repair mechanisms of the brain, immune and inflammatory reactions, and noninvasive brain imaging. In the first of these contributions, Emsley and collaborators discuss the possibility that cells generated from the stem cells of the adult brain could be mobilized for repair instead of transplanted cells. Widner and Barker give an overview of immune mechanisms and inflammatory responses associated with allogeneic and xenogeneic transplants in the CNS, which are important factors determining the survival and function not only of transplanted cells but also the regulation of endogenous neurogenesis and the survival of cells generated from endogenous precursors. In another paper, Brooks discusses the use of brain imaging to monitor graft survival and function. Noninvasive imaging by positron emission tomography, single-photon emission computed tomography, or magnetic resonance imaging provides essential tools for the visualization of the grafted cells and their survival, growth, and migration, and they are invaluable for patient selection, targeting transplants to specific areas, and for monitoring regenerative processes and functional changes after cell therapy. Finally, Cesaro gives a personal view on how to design clinical trials with cell therapy.

For the development of cell therapy for severe neurological diseases, it is important to make sure that the path to the clinic is guided by scientific progress, and that potential risks and benefits are properly assessed before any clinical trial. It is true that for many neurological and neurodegenerative disease states, the animal models available today are not perfect. Progress toward clinical trials must nevertheless be made with great care and with the support of convincing preclinical data, based on unequivocal proof of efficacy obtained in the best available animal models. Given time, cell-based therapies hold great promise for the future. However, the biological mechanisms underlying cell differentiation and repair are formidably complex. For this reason alone, progress in the cell therapy field is bound to be slow and made in steps, based on a gradual increase of our understanding of the cellular and molecular mechanisms involved.

Olle Lindvall E-mail: olle.lindvall@neurol.lu.se

Anders Björklund E-mail: anders.bjorklund@mphy.lu.se Guest Editors 